PROCEEDINGS OF THE

AMERICAN MATHEMATICAL SOCIETY

Volume 129, Number 7 , Pages 2069-2074

S 0002-9939(00)05842-

Article electronically published on November 30, 2000

\title{
DOUGLAS ALGEBRAS \\ WHICH ADMIT CODIMENSION 1 LINEAR ISOMETRIES
}

\author{
KEIJI IZUCHI
}

(Communicated by Joseph A. Ball)

\begin{abstract}
Let $B$ be a Douglas algebra and let $B_{b}$ be its Bourgain algebra. It is proved that $B$ admits a codimension 1 linear isometry if and only if $B \neq B_{b}$. This answers the conjecture of Araujo and Font.
\end{abstract}

\section{INTRODUCTION}

Let $H^{\infty}$ be the Banach algebra of bounded analytic functions on the unit disk $D$. Identifying a function in $H^{\infty}$ with its boundary function, we view $H^{\infty}$ as the closed subalgebra of $L^{\infty}$, the usual Lebesgue space on the unit circle $\partial D$. A closed subalgebra $B$ with $H^{\infty} \subset B \subset L^{\infty}$ is called a Douglas algebra. In [1], Araujo and Font studied codimension 1 linear isometries of Douglas algebras. They noted that $H^{\infty}$ has the codimension 1 linear isometry; $T f=z f, f \in H^{\infty}$, where $z$ is the identity function on $D$, and $L^{\infty}$ does not have any codimension 1 linear isometries, (see also [2]). They also gave the conjecture that proper Douglas algebras admit no codimension 1 linear isometries. In this paper, we give a characterization of Douglas algebras which admit codimension 1 linear isometries.

First, recall the structure of Douglas algebras. For a Douglas algebra $B$, we denote by $M(B)$ the set of non-zero multiplicative linear functionals of $B$. We consider the weak*-topology on $M(B)$. We identify a function in $B$ with its Gelfand transform. Then we may think of $M(B)$ as a compact subset of $M\left(H^{\infty}\right)$. It is known that $M\left(L^{\infty}\right)$ is the Shilov boundary of $H^{\infty}$. For a subset $E$ of $M\left(H^{\infty}\right)$, we denote by $\bar{E}$ the closure of $E$ in $M\left(H^{\infty}\right)$. For a function $f$ in $B$, let $Z_{B}(f)=\{x \in$ $M(B) ; f(x)=0\}$. For $x \in M\left(H^{\infty}\right)$, there is a unique probability measure $\mu_{x}$ on $M\left(L^{\infty}\right)$ such that $f(x)=\int_{M\left(L^{\infty}\right)} f d \mu_{x}$ for every $f \in H^{\infty}$. We denote by $\operatorname{supp} \mu_{x}$ the closed support set of $\mu_{x}$.

A function $f \in H^{\infty}$ is called inner if $|f|=1$ on $M\left(L^{\infty}\right)$. Let $\left\{z_{n}\right\}_{n}$ be a sequence in $D$ with $\sum_{n=1}^{\infty}\left(1-\left|z_{n}\right|\right)<\infty$. Then there is the associated Blaschke product $b$ given by

$$
b(z)=\prod_{n=1}^{\infty} \frac{-\bar{z}_{n}}{\left|z_{n}\right|} \frac{z-z_{n}}{1-\bar{z}_{n} z}, \quad z \in D .
$$

Received by the editors November 15, 1999.

2000 Mathematics Subject Classification. Primary 46J15, 47B38.

Supported by Grant-in-Aid for Scientific Research (No.10440039), Ministry of Education, Science and Culture. 
A sequence $\left\{z_{n}\right\}_{n}$ in $D$ is called interpolating if for every bounded sequence of complex numbers $\left\{a_{n}\right\}_{n}$ there exists $h \in H^{\infty}$ such that $h\left(z_{n}\right)=a_{n}$ for every $n$. A Blaschke product is called interpolating if its zero sequence is interpolating. It is known that a Blaschke product is inner (see Hoffman's book [10]).

The smallest Douglas algebra except $H^{\infty}$ is $H^{\infty}+C$, where $C$ is the space of continuous functions on $\partial D$, and it is known that $M\left(H^{\infty}+C\right)=M\left(H^{\infty}\right) \backslash D$ (see [14]). Chang [4] and Marshall [13] proved that if $B$ is a Douglas algebra, then $B=$ $H^{\infty}\left[\bar{b}_{\alpha} ; \alpha \in \Lambda\right]$, where $\left\{b_{\alpha}\right\}_{\alpha \in \Lambda}$ is a family of some interpolating Blaschke products and $M(B)=\bigcap_{\alpha \in \Lambda}\left\{x \in M\left(H^{\infty}\right) ;\left|b_{\alpha}(x)\right|=1\right\}$. And they showed that for Douglas algebras $A$ and $B$, it holds that $A \subset B$ if and only if $M(B) \subset M(A)$. By Chang and Marshall's theorem, we have that $x \in M(B)$ if and only if $B_{\mid \operatorname{supp} \mu_{x}}=\left(H^{\infty}\right)_{\mid \operatorname{supp} \mu_{x}}$. Put

$$
B_{\text {supp } \mu_{x}}=\left\{f \in L^{\infty} ; f_{\mid \operatorname{supp} \mu_{x}} \in B_{\mid \operatorname{supp} \mu_{x}}\right\} .
$$

Then $B_{\operatorname{supp} \mu_{x}}$ is a Douglas algebra and

$$
M\left(B_{\operatorname{supp} \mu_{x}}\right)=M\left(L^{\infty}\right) \cup\left\{y \in M(B) ; \operatorname{supp} \mu_{y} \subset \operatorname{supp} \mu_{x}\right\} .
$$

A nice reference for Douglas algebras is Garnett's book [6].

Next, recall the work of Hoffman [11]. For $x, y \in M\left(H^{\infty}\right)$, put

$$
\rho(x, y)=\sup \left\{|f(y)| ; f \in H^{\infty}, f(x)=0,\|f\|_{\infty} \leq 1\right\} .
$$

For $x \in M\left(H^{\infty}\right)$, let $P(x)=\left\{y \in M\left(H^{\infty}\right) ; \rho(x, y)<1\right\}$. When $P(x)=\{x\}$, a point $x$ is called trivial. When $P(x) \neq\{x\}$, in this case $x$ is called non-trivial; there is a one-to-one continuous map $L_{x}$ from $D$ onto $P(x)$ such that $f \circ L_{x} \in H^{\infty}$ for every $f \in H^{\infty}$ and $L_{x}(0)=x$. Moreover if $f(x)=0$, we can define the order of zero at $x$, ord $(f, x)$, by the usual order of zero of $f \circ L_{x}$ at $z=0$. When $f=0$ on $P(x)$, we put ord $(f, x)=\infty$.

For a Douglas algebra $B$, we denote by $B_{b}$ the set of $f$ in $L^{\infty}$ such that if $f_{n} \rightarrow 0$ weakly in $B$, then $\left\|f f_{n}+B\right\| \rightarrow 0$. Cima and Timoney [5] studied $B_{b}$ in a general setting. By their results, $B_{b}$ is a Douglas algebra and $B \subset B_{b}$. They called $B_{b}$ the Bourgain algebra of $B$. Douglas algebras $B$ satisfying $B \neq B_{b}$ are characterized in [7]. In this paper, we prove the following theorem.

Theorem. Let $B$ be a Douglas algebra. Then $B$ admits a codimension 1 linear isometry $T$ if and only if $B_{b} \neq B$. In this case $T$ has the following form: $T f=$ $(u b)(f \circ \varphi), f \in B$, where $u$ is an invertible unimodular function in $B, b$ is an interpolating Blaschke product, and $\varphi$ is a homeomorphism of $M\left(L^{\infty}\right)$ satisfying the following conditions.

(i) $B \circ \varphi=B$.

(ii) $\{y \in M(B) ;|b(y)|<1\}=P\left(x_{0}\right)$ for some $x_{0} \in M(B)$.

\section{Proof of the theorem}

First of all, we show a counterexample for Araujo and Font's conjecture. A Blaschke product $b$ with zeros $\left\{z_{n}\right\}_{n}$ is called sparse (or thin) if

$$
\lim _{n \rightarrow \infty} \prod_{j: j \neq n}\left|\frac{z_{j}-z_{n}}{1-\bar{z}_{n} z_{j}}\right|=1 \text {. }
$$

Let $x_{0} \in Z_{H^{\infty}+C}(b)$ and $B=H_{\operatorname{supp} \mu_{x_{0}}}^{\infty}$. Then $\{y \in M(B) ;|b(y)|<1\}=P\left(x_{0}\right)$ and $b B=\left\{g \in B ; g\left(x_{0}\right)=0\right\}$ (see [9]). Hence $T f=b f, f \in B$, is a codimension 1 linear isometry on $B$. 
To prove our theorem, we need two lemmas.

Lemma 1. Let $A$ and $B$ be Douglas algebras with $B \subsetneq A$. Then $M(B) \backslash M(A)$ is not a closed subset of $M(B)$.

Proof. Since $B \subsetneq A$, by Chang and Marshall's theorem $M(A) \subset M(B)$ and $M(B) \backslash$ $M(A) \neq \emptyset$. To prove our assertion, suppose not. Then $M(B) \backslash M(A)$ is an openclosed subset of $M(B)$. Hence by Shilov's idempotent theorem, there is $\chi \in B$ such that $\chi=1$ on $M(B) \backslash M(A)$ and $\chi=0$ on $M(A)$. Since $M\left(L^{\infty}\right) \subset M(A)$, $\chi=0$ on $M\left(L^{\infty}\right)$. Thus we get $\chi=0$ on $M(B)$ and $M(B) \backslash M(A)=\emptyset$. This is a contradiction.

Lemma 2. Let $A$ and $B$ be Douglas algebras with $B \subsetneq A$. Then

$$
M(A) \cap \overline{M(B) \backslash M(A)}
$$

contains uncountably many distinct points.

Proof. By Chang and Marshall's theorem, there is an interpolating Blaschke product $q$ such that

$$
\bar{q} \in A \quad \text { and } \quad \bar{q} \notin B .
$$

Then

$$
\emptyset \neq\{y \in M(B) ;|q(y)|<1\} \subset M(B) \backslash M(A) .
$$

We have

$$
q(M(B))=D \cup \partial D .
$$

For, if $\zeta \in D$, then $q_{\zeta}=(q-\zeta) /(1-\bar{\zeta} q)$ is an inner function and $q_{\zeta}$ is not invertible in $B$. Hence there is $x \in M(B)$ such that $q_{\zeta}(x)=0$. Thus we get $\zeta \in q(M(B))$.

Let

$$
\Gamma=\overline{\{y \in M(B) ;|q(y)|<1\}} \backslash\{y \in M(B) ;|q(y)|<1\} .
$$

Then by (2.3), we have $q(\Gamma)=\partial D$. For each $\xi \in \partial D$, take $x_{\xi} \in \Gamma$ such that $q\left(x_{\xi}\right)=\xi$. Then $q=\xi$ on $\operatorname{supp} \mu_{x_{\xi}}$ and

$$
\operatorname{supp} \mu_{x_{\xi}} \cap \operatorname{supp} \mu_{x_{\eta}}=\emptyset \quad \text { if } \xi, \eta \in \partial D \text { and } \xi \neq \eta \text {. }
$$

We shall prove that for each $\xi \in \partial D$ there exists $y_{\xi} \in M(B)$ such that

$$
\operatorname{supp} \mu_{y_{\xi}} \subset \operatorname{supp} \mu_{x_{\xi}} \quad \text { and } \quad y_{\xi} \in M(A) \cap \overline{M(B) \backslash M(A)} .
$$

Then by (2.5), points in $\left\{y_{\xi} ; \xi \in \partial D\right\}$ are distinct and we get our assertion.

We shall show the existence of $y_{\xi}$ satisfying (2.6). If $x_{\xi} \in M(A)$, by (2.1) we have $\left|q\left(x_{\xi}\right)\right|=1$. Since $x_{\xi} \in \Gamma$, by $(2.4)$ we have $x_{\xi} \in \overline{\{y \in M(B) ;|q(y)|<1\}}$. Hence by $(2.2), x_{\xi} \in \overline{M(B) \backslash M(A)}$. Thus $x_{\xi} \in M(A) \cap \overline{M(B) \backslash M(A)}$. Put $y_{\xi}=x_{\xi}$; then (2.6) holds.

Next, suppose that $x_{\xi} \notin M(A)$. Put $A_{1}=A_{\text {supp } \mu_{x_{\xi}}}$ and $B_{1}=B_{\text {supp } \mu_{x_{\xi}}}$. Then $B_{1} \subset A_{1}$. Since $x_{\xi} \in M(B), B_{1}=\left(H^{\infty}\right)_{\operatorname{supp} \mu_{x_{\xi}}}$. Since $x_{\xi} \notin M(B), A_{1} \neq$ $\left(H^{\infty}\right)_{\text {supp } \mu_{x_{\xi}}}$. Hence we have $B_{1} \neq A_{1}$. By Lemma 1 , there exists a point $y_{\xi}$ such that

$$
y_{\xi} \in M\left(A_{1}\right) \cap \overline{M\left(B_{1}\right) \backslash M\left(A_{1}\right)} .
$$


We have

$$
\begin{aligned}
& M\left(B_{1}\right)=M\left(L^{\infty}\right) \cup\left\{y \in M(B) ; \operatorname{supp} \mu_{y} \subset \operatorname{supp} \mu_{x_{\xi}}\right\}, \\
& M\left(A_{1}\right)=M\left(L^{\infty}\right) \cup\left\{y \in M(A) ; \operatorname{supp} \mu_{y} \subset \operatorname{supp} \mu_{x_{\xi}}\right\} .
\end{aligned}
$$

By the above

$$
M\left(B_{1}\right) \backslash M\left(A_{1}\right)=\left\{y \in M(B) \backslash M(A) ; \operatorname{supp} \mu_{y} \subset \operatorname{supp} \mu_{x_{\xi}}\right\} .
$$

Hence by (2.7), we have supp $\mu_{y_{\xi}} \subset \operatorname{supp} \mu_{x_{\xi}}$. Since $M\left(A_{1}\right) \subset M(A)$ and by (2.7) and (2.8), we have $y_{\xi} \in M(A) \cap \overline{M(B) \backslash M(A)}$.

Proof of the theorem. Suppose that $B_{b} \neq B$. By Chang and Marshall's theorem, there is an interpolating Blaschke product $\psi$ such that $\bar{\psi} \in B_{b}$ and $\bar{\psi} \notin B$. By 7. Theorem 2], $Z_{B}(\psi)$ is a finite set. Let $Z_{B}(\psi)=\left\{x_{1}, x_{2}, \ldots, x_{n}\right\}$ such that $x_{i} \neq x_{j}, i \neq j$. Take an open subset $V$ of $M\left(H^{\infty}\right)$ such that $x_{1} \in V$ and $x_{j} \notin \bar{V}$ for $j=2,3, \ldots, n$. Let $\left\{z_{n}\right\}_{n}$ be the zeros of $\psi$ in $D$. Let $\psi_{1}$ be the subproduct of $\psi$ whose zeros are $\left\{z_{n}\right\}_{n} \cap V$. By [10, p. 205], $Z_{H^{\infty}}(\psi)=\overline{\left\{z_{n}\right\}_{n}}$, so that we have $Z_{B}\left(\psi_{1}\right)=\left\{x_{1}\right\}$. Let $T f=\psi_{1} f$ for $f \in B$. Then $T$ is a linear isometry on $B$ and $T B \subset\left\{g \in B ; g\left(x_{1}\right)=0\right\}$. By [3, 9, for $g \in B$ with $g\left(x_{1}\right)=0$ there exists $h \in B$ such that $g=\psi_{1} h$. Hence $T B=\left\{g \in B ; g\left(x_{1}\right)=0\right\}$, so that $T$ is a codimension 1 linear isometry on $B$.

Suppose that $B$ admits a codimension 1 linear isometry $T$. Then by the work of Araujo and Font [1, there exist a homeomorphism $\varphi$ of $M\left(L^{\infty}\right)$ and a unimodular function $\psi$ on $M\left(L^{\infty}\right)$ such that

$$
(T f)(x)=\psi(x) f(\varphi(x)) \quad \text { for all } x \in M\left(L^{\infty}\right) \text { and } f \in B .
$$

Since $1 \in B$, we have

$$
\psi \in B
$$

First, we prove that

$$
B \circ \varphi \subset B .
$$

To prove this, suppose not. Let $A$ be the Douglas algebra generated by $B$ and $B \circ \varphi$. Then $B \subsetneq A$. By (2.9) and (2.10), we have $\psi A \subset B$. Hence by [15, Theorem 1],

$$
M(B) \backslash M(A) \subset Z_{B}(\psi) .
$$

By Lemma 2, $M(A) \cap \overline{(M(B) \backslash M(A))}$ is an uncountable set. Let $y \in M(A) \cap$ $\overline{(M(B) \backslash M(A))}$ and $f \in B$. Since $\psi, f \circ \varphi \in A$ and $y \in M(A)$, we have $(T f)(y)=$ $(\psi f \circ \varphi)(y)=\psi(y)(f \circ \varphi)(y)$. Since $y \in \overline{M(B) \backslash M(A)}$, by $(2.12) \psi(y)=0$. Hence $(T f)(y)=0$, so that

$$
M(A) \cap \overline{(M(B) \backslash M(A))} \subset Z_{A}(T f) \subset Z_{B}(T f) \text { for every } f \in B .
$$

By [1, Proposition 3.1], $\bigcap\left\{Z_{B}(T f) ; f \in B\right\}$ is a finite set. Hence by the above, $M(A) \cap \overline{(M(B) \backslash M(A))}$ is a finite set. This is a contradiction. Thus we obtain $(2.11)$.

Next, we prove that

$$
\bar{\psi} \notin B .
$$

To prove this, suppose not. Then $\bar{\psi} \in B$ and $\psi$ is invertible in $B$. Put $T_{0} f=\bar{\psi} T f$ for $f \in B$. Then $T_{0}$ is a codimension 1 linear isometry on $B$. By $(2.9), T_{0} B=B \circ \varphi$ 
and there exists $\lambda \in B$ such that $B=B \circ \varphi+\mathbf{C} \lambda$ and $\lambda \notin B \circ \varphi$, where $\mathbf{C}$ is the set of complex numbers. Then we have

$$
B \circ \varphi^{-1}=B+\mathbf{C} \lambda \circ \varphi^{-1} \text { and } \lambda \circ \varphi^{-1} \notin B .
$$

Since $\varphi$ is a homeomorphism of $M\left(L^{\infty}\right), B \circ \varphi^{-1}$ is a closed subalgebra of $L^{\infty}$ and $H^{\infty} \subset B$. Then by the above, $B \circ \varphi^{-1}$ is a Douglas algebra which contains $B$ properly. And $B$ is a linear subspace of $B \circ \varphi^{-1}$ of codimension 1. By Chang and Marshall's theorem, there exists an interpolating Blaschke product $b$ such that $\bar{b} \in B \circ \varphi^{-1}$ and $\bar{b} \notin B$. Since $\bar{b}^{n} \in B \circ \varphi^{-1} \backslash B$ and $\left\{\bar{b}^{n} ; n=1,2, \ldots\right\}$ is linearly independent, $B$ is not a linear subspace of $B \circ \varphi^{-1}$ of codimension 1 . This is a contradiction. Thus we get (2.13).

By (2.13), there exists $x_{0} \in M(B)$ such that $\psi\left(x_{0}\right)=0$. By (2.9), (2.10), and (2.11), $T f=0$ on $Z_{B}(\psi)$ for every $f \in B$. Then we have

$$
Z_{B}(\psi)=\left\{x_{0}\right\} .
$$

For, if $y \in Z_{B}(\psi)$ and $y \neq x_{0}$, there exist $f, g \in B$ such that $f(y)=1, f\left(x_{0}\right)=0$, and $g(y)=0, g\left(x_{0}\right)=1$. Then $f, g \notin T B$ and $f, g$ are linear independent. Hence $T$ is not a codimension 1 linear isometry.

Also we have

$$
T B=\left\{f \in B ; f\left(x_{0}\right)=0\right\}
$$

since $T B \subset\left\{f \in B ; f\left(x_{0}\right)=0\right\}$ and $T B$ is a linear subspace of $B$ of codimension 1 .

Then we have

$$
\begin{aligned}
\psi B & \subset & \left\{f \in B ; f\left(x_{0}\right)=0\right\} & \text { by }(2.14) \\
& =\psi B \circ \varphi & \text { by }(2.9) & \text { and }(2.15) \\
& \subset \psi B & \text { by }(2.11) . &
\end{aligned}
$$

Thus we get

$$
\begin{gathered}
B=B \circ \varphi, \\
\psi B=\left\{f \in B ; f\left(x_{0}\right)=0\right\} .
\end{gathered}
$$

We prove that $B \neq B_{b}$. By (2.13), it is sufficient to prove $\bar{\psi} \in B_{b}$. To show this, let $\left\{f_{n}\right\}_{n} \subset B$ such that $f_{n} \rightarrow 0$ weakly in $B$. Then $f_{n}\left(x_{0}\right) \rightarrow 0$. By (2.17), $f_{n}-f_{n}\left(x_{0}\right) \in \psi B$. Then there exists $g_{n} \in B$ such that $f_{n}-f_{n}\left(x_{0}\right)=\psi g_{n}$. Hence we have $\left\|f_{n} \bar{\psi}-B\right\| \leq\left\|f_{n} \bar{\psi}-g_{n}\right\| \leq\left|f_{n}\left(x_{0}\right)\right| \rightarrow 0$ as $n \rightarrow \infty$. Therefore $\bar{\psi} \in B_{b}$.

Now we complete the proof. Condition (i) follows from (2.16). Since $x_{0} \in$ $M(B), B_{\mid \operatorname{supp} \mu_{x_{0}}}=\left(H^{\infty}\right)_{\mid \operatorname{supp} \mu_{x_{0}}}$. By $(2.10)$, there exists $h \in H^{\infty}$ such that $h_{\mid \operatorname{supp} \mu_{x_{0}}}=\psi_{\mid \operatorname{supp} \mu_{x_{0}}}$. If ord $\left(h, x_{0}\right) \geq 2$, there is a factorization $h=h_{1} h_{2}$ such that $h_{1}\left(x_{0}\right)=h_{2}\left(x_{0}\right)=0$ and $h_{1}, h_{2} \in H^{\infty}$ (see [11, Section 5]). Since $h_{i} \in B$, by (2.17) we have $h_{i}=\psi g_{i}$ for some $g_{i} \in B$ for $i=1,2$. Then $h=\psi^{2} g_{1} g_{2}$. Hence $\psi_{\mid \operatorname{supp} \mu_{x_{0}}}=\left(\psi^{2} g_{1} g_{2}\right)_{\mid \operatorname{supp} \mu_{x_{0}}}$. Since $|\psi|=1$ on $M\left(L^{\infty}\right), 1=\left(\psi g_{1} g_{2}\right)_{\mid \operatorname{supp} \mu_{x_{0}}}$. Since $\left(\psi g_{1} g_{2}\right)\left(x_{0}\right)=0$, we have a contradiction. Hence $\operatorname{ord}\left(h, x_{0}\right)=1$ and $x_{0}$ is a nontrivial point. Therefore by (2.14) and [12, Corollary 4.5], there are an interpolating Blaschke product $b$ and an invertible unimodular function $u$ in $B$ such that $\psi=u b$ and $Z_{B}(b)=\left\{x_{0}\right\}$. Then by [8, Theorem 3.2], $\{y \in M(B) ;|b(y)|<1\}=P\left(x_{0}\right)$. This completes the proof. 


\section{REFERENCES}

[1] J. Araujo and J. J. Font, Codimension 1 linear isometries on function algebras, Proc. Amer. Math. Soc. 127(1999), 2273-2281. MR 99j:46059

[2] J. Araujo and J. J. Font, Linear isometries between subspaces of continuous functions, Trans. Amer. Math. Soc. 349(1997), 413-428. MR 97d:46026

[3] S. Axler and P. Gorkin, Division in Douglas algebras, Michigan Math. J. 31(1984), 89-94. MR 85h: 46075

[4] S.-Y. Chang, A characterization of Douglas algebras, Acta Math. 137(1976), 81-89. MR 55:1074a

[5] J. Cima and R. Timoney, The Dunford Pettis property for certain planner uniform algebras, Michigan Math. J. 34(1987), 99-104. MR 88e:46023

[6] J. Garnett, Bounded Analytic Functions, Academic Press, New York, 1981. MR 83g:30037

[7] P. Gorkin, K. Izuchi, and R. Mortini, Bourgain algebras of Douglas algebras, Canad. J. Math. 44(1992), 797-804. MR 94c:46104

[8] P. Gorkin, H. -M. Lingenberg, and R. Mortini, Homeomorphic disks in the spectrum of $H^{\infty}$, Indiana Univ. Math. J. 39(1990), 961-983. MR 92b:46082

[9] C. Guillory, K. Izuchi and D. Sarason, Interpolating Blaschke products and division in Douglas algebras, Proc. Roy. Irish Acad. Sect. A 84(1984), 1-7. MR 86j:46054

[10] K. Hoffman, Banach Spaces of Analytic Functions, Prentice Hall, Englewood Cliffs, N.J., 1962. MR 24:A2844

[11] K. Hoffman, Bounded analytic functions and Gleason parts, Michigan Math. J. 40(1993), 53-75.

[12] K. Izuchi, Interpolating Blaschke products and factorization theorems, J. London Math. Soc. (2) 50(1994), 547-567. MR 95k:46086

[13] D. Marshall, Subalgebras of $L^{\infty}$ containing $H^{\infty}$, Acta Math. 137(1976), 91-98. MR 55:10746

[14] D. Sarason, Algebras of functions on the unit circle, Bull. Amer. Math. Soc. 79(1973), 286299. MR 48:2777

[15] R. Younis, Division in Douglas algebras and some applications, Arch. Math. 45(1985), 555560. MR 87b:46059

Department of Mathematics, Niigata University, Nimgata 950-2181, Japan

E-mail address: izuchi@math.sc.niigata-u.ac.jp 